\title{
Computational fluid-structure interaction: methods and application to a total cavopulmonary connection
}

\author{
Yuri Bazilevs · M.-C. Hsu • D. J. Benson • \\ S. Sankaran - A. L. Marsden
}

Received: 9 August 2009 / Accepted: 8 September 2009 / Published online: 24 September 2009

(c) The Author(s) 2009. This article is published with open access at Springerlink.com

\begin{abstract}
The Fontan procedure is a surgery that is performed on single-ventricle heart patients, and, due to the wide range of anatomies and variations among patients, lends itself nicely to study by advanced numerical methods. We focus on a patient-specific Fontan configuration, and perform a fully coupled fluid-structure interaction (FSI) analysis of hemodynamics and vessel wall motion. To enable physiologically realistic simulations, a simple approach to constructing a variable-thickness blood vessel wall description is proposed. Rest and exercise conditions are simulated and rigid versus flexible vessel wall simulation results are compared. We conclude that flexible wall modeling plays an important role in predicting quantities of hemodynamic interest in the Fontan connection. To the best of our knowledge, this paper presents the first three-dimensional patientspecific fully coupled FSI analysis of a total cavopulmonary connection that also includes large portions of the pulmonary circulation.
\end{abstract}

Keywords Blood flow · Fontan surgery · Fluid-structure interaction · Variable wall thickness · Hyperelasticity .

Wall shear stress

\section{Introduction}

Congenital heart defects are among the most prevalent form of birth defects, occurring in roughly $1 \%$ of births. "Single

\section{Y. Bazilevs $(\varangle) \cdot$ M.-C. Hsu · D. J. Benson}

Department of Structural Engineering, University of California, San Diego, 9500 Gilman Drive, La Jolla, CA 92093, USA

e-mail: yuri@ucsd.edu

\section{S. Sankaran · A. L. Marsden}

Department of Mechanical and Aerospace Engineering,

University of California, San Diego, 9500 Gilman Drive,

La Jolla, CA 92093, USA ventricle"-type defects refer to cases where the heart has only one effective or functional pumping chamber, and are usually fatal shortly after birth if left untreated. Single ventricle patients usually require a staged surgical approach which culminates with a Fontan procedure [13]. There are two variants of the Fontan procedure, the extra-cardiac conduit (ECC) and the lateral tunnel (LT) [31]. In both cases, the superior vena cava (SVC) is connected to the right pulmonary artery. In the ECC variant, a baffle is also constructed to connect the inferior vena cava (IVC) to the pulmonary arteries, resulting in a modified T-shaped junction. In the LT variant, a tunnel-like patch is placed inside the atrium so that blood returning from the inferior vena cava is directed through this tunnel. A connection is then made between the end of the tunnel/top of the right atrium and the underside of the pulmonary artery. As a result of both the ECC and the LT, the circulation becomes a single pump system, and the heart contains only oxygenated blood. A surgical connection of the SVC and IVC directly to the left and right pulmonary arteries is referred to as the total cavopulmonary connection.

Congenital heart disease is a field that lends itself well to study by numerical techniques due to the wide range of anatomies and variations among patients. Numerical techniques allow us to examine the effects of the geometry of the Fontan connection that plays an important role in the overall success of the surgery, and assess blood flow characteristics and energy losses associated with a given surgical design. There are numerous articles on the total cavopulmonary connection simulation (see, e.g., $[7,11,22,25,26]$ ) that do a very careful CFD analysis on complex patient-specific configurations in an attempt to answer some of these questions. Some of the earlier work in computational fluid dynamics applied to congenital heart disease compared energy loss in the standard " $\mathrm{t}$ " junction Fontan with the proposed "offset" model, and led to the adoption of the offset model as the currently preferred 
method $[9,10,28,29]$. However, the authors feel that despite the abundance of articles on the subject, very little clinical impact on Fontan surgery has been derived directly from simulations. This is in part attributable to the limitations of the simulation methods used for this application. This article addresses one of these shortcoming by introducing flexible wall modeling in Fontan surgery simulations. Including arterial fluid-structure interaction (FSI) has been found to be important for modeling other parts of the cardiovascular system (see, e.g., [5,46,47,51]), and the Fontan surgery, as will be shown in this article, presents no exception. It should be noted that an idealized Fontan configuration without pulmonary branching was studied using flow-structure interaction in [27].

The paper is outlined as follows. In Sect. 2, a simple approach to vessel wall thickness reconstruction is proposed. Modeling and discretization of the blood vessel wall requires information not only about the material properties, but also local wall thickness. In previous work, the wall thickness is often directly related to the vessel radius and is expressed in terms of its percentage. This definition is useful for vessels that are straight and circular, however, its meaning is lost for the case of real patient-specific vasculature due to the presence of local curvature, vessel branching, and geometric anomalies, such as aneurysms. CT imaging is able to produce accurate blood volume data, yet the wall thickness information is not easily accessible. The proposed method for vessel wall thickness construction may be employed if the only information available to the analyst is a mesh of the vessel volume occupied by the blood. This is often the case when commercial mesh generation packages are employed that currently do not have a built-in capability to generate solid layer meshes. The method we present in this work is based on solving the Laplace partial differential equation to prescribe the thickness and is able to handle arbitrarily complex geometries.

In Sect. 3, a structural model of the vessel wall that only make use of displacement degrees of freedom and does not require a full three-dimensional solid discretization is described. This approach is adopted due to the fact that threedimensional vessel wall meshing is currently not available to us for the application presented here. However, for the purposes of presenting ideas in this article, this modeling approach is deemed sufficient. We expect to improve it in the near future by employing a complete shell formulation with rotational degrees of freedom or developing meshing techniques in support of three-dimensional solid modeling.

In Sect. 4, numerical results for an ECC variant of a patient-specific Fontan surgery configuration are presented. Rest and exercise conditions are simulated and rigid and flexible vessel wall modeling approaches are compared. The results show that rigid wall modeling gives an overestimation of the wall shear stress and pressure, especially for the case of exercise conditions. It is also shown that the resistance boundary condition employed in the computations and enforced only weakly at each outlet actually holds in a nearly strong sense.

In Sect. 5, conclusions and future work are presented.

\section{Vessel wall thickness reconstruction}

Let $\Omega \in \mathcal{R}^{3}$ be the blood vessel domain occupied by the blood, and $\Gamma$ be its boundary. Let $\Gamma_{i}, i=1,2, \ldots, n_{s r f}$, denote $i$ th inlet or outlet surface, and $n_{s r f}$ be the total number of inlets and outlets in a given patient-specific model. We introduce a volumetric thickness function $T: \Omega \rightarrow \mathcal{R}$ whose restriction to the arterial surface approximates the actual thickness of the arterial wall. The thickness function $T$ is assumed to satisfy the following boundary value problem:

$$
\begin{aligned}
& -\Delta T=0 \text { in } \Omega \\
& T=\left(\frac{\int_{\Gamma_{i}} d \Gamma}{\pi}\right)^{1 / 2} \times x \% \text { on } \Gamma_{i} \\
& \frac{\partial T}{\partial n}=0 \text { on } \Gamma \backslash \bigcup_{i=1}^{n_{s r f}} \Gamma_{i}
\end{aligned}
$$

The formulation corresponds to the Laplace equation for $T$ subject to prescribed Dirichlet boundary conditions at inlets and outlets, where $x$ is the wall thickness expressed as a percentage of the area-averaged radius. Homogeneous Neumann or flux boundary conditions are assumed to hold on the remainder of the boundary.

The method effectively collects the wall thickness information at the inlets and outlets of the patient-specific model and propagates it into the domain interior. Smooth distribution of wall thickness is expected everywhere in the domain, including geometrically complex branching regions, due to the favorable properties of the Laplace operator. The method can be applied to any patient-specific model independent of its complexity, and guarantees that the wall thickness at all inlets and outlets is exactly $x \%$ of the area-averaged radius. The formulation (1)-(3) is amenable to a heat transfer interpretation, where inlets and outlets correspond to regions of prescribed temperature with no heat exchange on the rest of the boundary.

We tested the proposed thickness reconstruction method on an idealized bifurcation model as well as patient-specific Fontan surgery configurations. In both cases, inlet and outlet vessel wall thickness was assumed to be $10 \%$ of the respective area-averaged radii. Figures 1 and 2 show the resultant wall thickness distribution for the bifurcation and Fontan models, respectively. In both cases a very reasonable smooth distribution of wall thickness is attained, especially considering how little information was taken as input data. In particular, the 


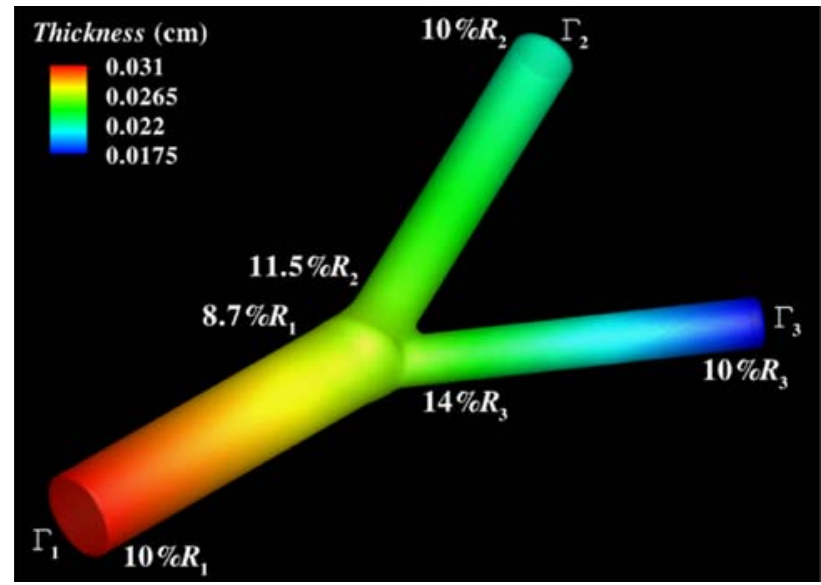

Fig. 1 Reconstructed thickness distribution from inlet and outlet data for an idealized bifurcation model. The radii of the arterial branches are $R_{1}=0.31 \mathrm{~cm}, R_{2}=0.22 \mathrm{~cm}$, and $R_{3}=0.175 \mathrm{~cm}$. Near the bifurcation, the largest branch thins to $8.7 \%$ of its radius, while the two smaller ones thicken to 11.5 and $14 \%$ of their respective radii

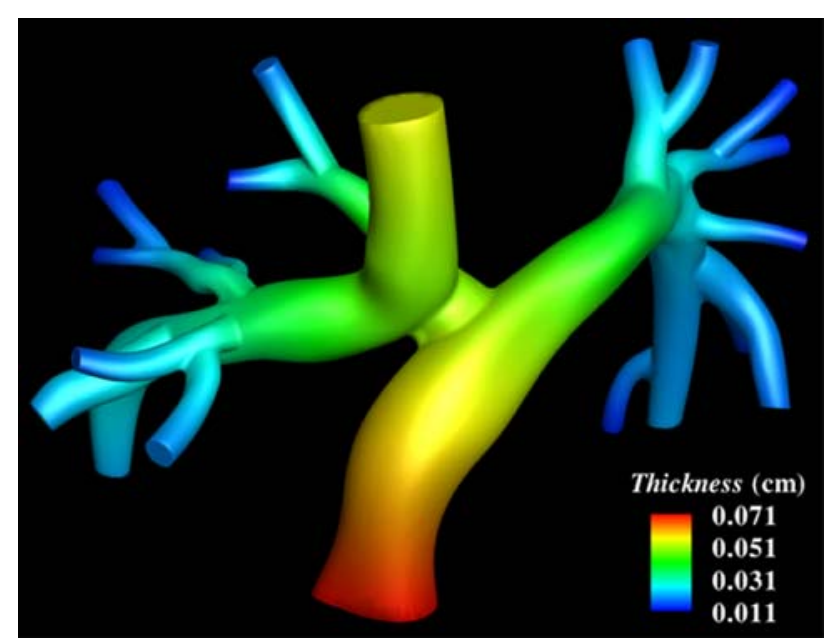

Fig. 2 Reconstructed thickness distribution from inlet and outlet data for a patient-specific Fontan surgery configuration

results of the Fontan configuration show a physiologically realistic, gradual thinning of the vessel wall from larger to smaller branches.

Remark It should be noted that the thickness boundary condition specification is not restricted to the inlets and outlets. This information, if available from measurements or other sources, may be incorporated in other parts of the patientspecific model domain. In situations where the geometry is locally complex (such as extreme stenosis or aneurysm), additional constraints on the thickness can be imposed at specific locations within the domain.

Remark In this paper we do not claim that vessel wall thickness is distributed according to the Laplace equation. The proposed method is an approximate technique that allows for incorporation of a reasonably realistic variable wall thickness in the simulations that make use of limited input data. This approach gives more physiologically realistic results than the constant wall thickness assumption, which is employed in most patient-specific vascular flow-structure interaction computations reported in the literature.

Remark We would like to note that there are a few recent variable wall thickness computations reported in [42] with a membrane wall model, in [37,49] with a continuum wall model, and in [36] with a continuum wall model and fairly complex arterial shapes. However, the technique used in this article is more general and easier to use than those employed in $[36,37,49]$.

\section{Vessel wall modeling}

\subsection{Kinematics}

Let $\boldsymbol{X}$ denote the coordinates of the reference or material configuration of the blood vessel. We assume that the vessel wall is discretized into three-node triangles and define its parameterization, restricted to each triangular element, as

$\boldsymbol{X}\left(\xi_{1}, \xi_{2}, \xi_{3}\right)=\sum_{A=1}^{3} N_{A}\left(\xi_{1}, \xi_{2}\right) \boldsymbol{X}_{A}+H \xi_{3} \boldsymbol{N}$

In the above equation, $\xi_{1}$ and $\xi_{2}$ are the surface or in-plane parametric coordinates, $\xi_{3}$ is the through-thickness parametric coordinate, $H$ is the vessel wall thickness in the reference configuration, $N_{A}$ 's are the triangular element shape functions, and $\boldsymbol{X}_{A}$ 's are the nodal coordinates of the luminal surface. Also in Eq. (4), $N$ is the unit outward normal in the reference configuration given by

$\boldsymbol{N}=\frac{\frac{\partial \boldsymbol{X}}{\partial \xi_{1}} \times \frac{\partial \boldsymbol{X}}{\partial \xi_{2}}}{\left\|\frac{\partial \boldsymbol{X}}{\partial \xi_{1}} \times \frac{\partial \boldsymbol{X}}{\partial \xi_{2}}\right\|}$.

Because we associate $\boldsymbol{X}_{A}$ 's with a luminal surface rather than the blood vessel mid-surface, the parametric coordinate $\xi_{3}$ is assumed to take values in the interval $[0,1]$. In equation (4), we also assumed that the vessel thickness $H$ is constant on every triangle, however, some thickness variation from triangle to triangle is expected.

The displacement field $\boldsymbol{u}$ is assumed to be a function of in-plane parametric coordinates only, and is given by

$\boldsymbol{u}\left(\xi_{1}, \xi_{2}\right)=\sum_{A} N_{A}\left(\xi_{1}, \xi_{2}\right) \boldsymbol{u}_{A}$,

where $\boldsymbol{u}_{A}$ 's are the nodal displacement degrees of freedom. The deformation gradient $\boldsymbol{F}$ becomes

$\boldsymbol{F}=\left(\boldsymbol{I}+\frac{\partial \boldsymbol{u}}{\partial \boldsymbol{X}}\right)$ 
for which the displacement gradient $\partial \boldsymbol{u} / \partial \boldsymbol{X}$ is computed as

$\frac{\partial \boldsymbol{u}}{\partial \boldsymbol{X}}=\left[\begin{array}{ccc}\uparrow & \uparrow & \uparrow \\ \frac{\partial \boldsymbol{u}}{\partial \xi_{1}} & \frac{\partial \boldsymbol{u}}{\partial \xi_{2}} & \mathbf{0} \\ \downarrow & \downarrow & \downarrow\end{array}\right]\left[\begin{array}{ccc}\uparrow & \uparrow & \uparrow \\ \frac{\partial \boldsymbol{X}}{\partial \xi_{1}} & \frac{\partial \boldsymbol{X}}{\partial \xi_{2}} & \frac{\partial \boldsymbol{X}}{\partial \xi_{3}} \\ \downarrow & \downarrow & \downarrow\end{array}\right]^{-1}$.

The additional kinematic quantities to be used in the sequel are $\boldsymbol{C}=\boldsymbol{F}^{T} \boldsymbol{F}$ and $J=\operatorname{det} \boldsymbol{F}$, the Cauchy-Green deformation tensor and the determinant of the deformation gradient, respectively (see, e.g., [16]).

\subsection{The weak formulation}

Let $\mathcal{V}^{h}$ and $\mathcal{W}^{h}$ be the discrete solution and weighting function spaces. The semi-discrete weak formulation of the solid problem is stated as follows: Find $\boldsymbol{u} \in \mathcal{V}^{h}$, such that $\forall \boldsymbol{w} \in$ $\mathcal{W}^{h}$

$$
\begin{gathered}
\sum_{e=1}^{N_{s e l}} \int_{\Omega_{e}} \boldsymbol{w} \rho_{0} \frac{\partial^{2} \boldsymbol{u}}{\partial t^{2}} d \Omega_{e}+\int_{\Omega_{e}} \nabla_{X} \boldsymbol{w}: \boldsymbol{F} \boldsymbol{S} d \Omega_{e} \\
-\int_{\Omega_{e}} \boldsymbol{w} \rho_{0} \boldsymbol{f} d \Omega_{e}-\int_{\Gamma_{e}} \boldsymbol{w} \boldsymbol{h} d \Gamma_{e}=0,
\end{gathered}
$$

where $\rho_{0}$ is the tissue density, $\boldsymbol{f}$ and $\boldsymbol{h}$ are the body and surface forces, respectively, $d \Gamma_{e}$ is the infinitesimal element of the shell surface, $d \Omega_{e}=H_{e} d \Gamma_{e}$, and $H_{e}$ is the thickness of the triangular element $e$. All quantities in the above formulation are referred to the reference configuration and the summation is taken over $N_{\text {sel }}$ triangular surface elements.

We model the tissue as a hyperelastic material and assume the existence of a stored elastic energy of the form

$\varphi(\boldsymbol{C}, J)=\frac{1}{2} \mu\left(J^{-2 / 3} \operatorname{tr} \boldsymbol{C}-3\right)+\frac{1}{2} \kappa\left(\frac{1}{2}\left(J^{2}-1\right)-\ln J\right)$.

From (10), the second Piola-Kirchhoff stress tensor $S$ and the fourth-rank tensor of material tangent moduli $\boldsymbol{C}$ are obtained by performing the following differentiations

$\boldsymbol{S}=2 \frac{\partial \varphi}{\partial \boldsymbol{C}}(\boldsymbol{C}, J)$

and

$\boldsymbol{C}=4 \frac{\partial^{2} \varphi}{\partial \boldsymbol{C} \partial \boldsymbol{C}}(\boldsymbol{C}, J)$.

Explicit expressions for $\boldsymbol{S}$ and $\boldsymbol{C}$ in terms of $\boldsymbol{C}$ and $\boldsymbol{J}$ are lengthy, so we do not present them here and refer the reader to [2] for details. Parameters $\mu$ and $\kappa$ in (10) are the material shear and bulk moduli, respectively.
3.3 Enforcement of zero through-thickness stress condition

To avoid thickness locking and ensure consistency with the three-dimensional theory, normal stress in the thickness direction must vanish (see, e.g, [6]). The zero throughthickness stress condition may be expressed as

$S_{N N} \equiv \boldsymbol{N} \cdot S \boldsymbol{N}=0$,

where $S_{N N}$ is a scalar value of the through-thickness stress. In the case of a linear stress-strain relationship, the enforcement of the above condition may be accomplished by appropriately modifying the tensor of elastic moduli (see,, e.g., [17]) and, as a result, the stress-strain law. In our case, the stress-strain relationship (11) is nonlinear, which motivates the following Newton iteration approach to satisfying equation (13). The idea is to linearize (13) and iterate on a through-thickness strain until convergence.

We first define a through-thickness strain component as

$C_{N N} \equiv N \cdot C N$

and a corresponding deformation gradient component as

$F_{n N} \equiv \boldsymbol{n} \cdot \boldsymbol{F N}$,

where $\boldsymbol{n}$ is the unit outward normal in the current configuration given by

$\boldsymbol{n}=\frac{\frac{\partial \mathbf{x}}{\partial \xi_{1}} \times \frac{\partial \mathbf{x}}{\partial \xi_{2}}}{\left\|\frac{\partial \mathbf{x}}{\partial \xi_{1}} \times \frac{\partial \mathbf{x}}{\partial \xi_{2}}\right\|}$,

and $\boldsymbol{x}$ 's are the coordinates of the current configuration given by

$x=X+u$

The following three-stage algorithm is executed at every integration point on the triangle surface (one point integration is used on the stress terms):

Stage 1-Initialization: Given the displacement gradient $\partial \boldsymbol{u} / \partial \boldsymbol{X}$, initialize

$\boldsymbol{F}^{(0)}=(\boldsymbol{I}+\partial \boldsymbol{u} / \partial \boldsymbol{X})$

$\boldsymbol{C}^{(0)}=\boldsymbol{F}^{(0) T} \boldsymbol{F}^{(0)}$

$J^{(0)}=\operatorname{det} \boldsymbol{F}^{(0)}$

$F_{n N}^{(0)}=\boldsymbol{n} \cdot \boldsymbol{F}^{(0)} \boldsymbol{N}$

$C_{N N}^{(0)}=\boldsymbol{N} \cdot \boldsymbol{C}^{(0)} \boldsymbol{N}$,

where the bracketed superscript denotes the iteration index.

Stage 2-Iteration: For $i=0,1, \ldots, n_{\max }$ repeat the following steps 
(1) Compute

$$
\begin{aligned}
& \boldsymbol{S}^{(i)}=2 \frac{\partial \varphi}{\partial \boldsymbol{C}}\left(\boldsymbol{C}^{(i)}, J^{(i)}\right) \\
& \boldsymbol{C}^{(i)}=4 \frac{\partial^{2} \varphi}{\partial \boldsymbol{C} \partial \boldsymbol{C}}\left(\boldsymbol{C}^{(i)}, \boldsymbol{J}^{(i)}\right) \\
& S_{N N}^{(i)}=\boldsymbol{N} \cdot \boldsymbol{S}^{(i)} \boldsymbol{N} \\
& \left(\frac{\partial S_{N N}}{\partial C_{N N}}\right)^{(i)}=\frac{1}{2} N_{I} N_{J} C_{I J K L}^{(i)} N_{K} N_{L},
\end{aligned}
$$

where $C_{I J K L}$ and $N_{I}$ are the cartesian components of $C$ and $N$, respectively.

(2) Solve for the increment $\Delta C_{N N}^{i+1}$ from the Newton linearization of the zero through-thickness stress condition (13)

$$
\left(\frac{\partial S_{N N}}{\partial C_{N N}}\right)^{(i)} \Delta C_{N N}^{(i+1)}+S_{N N}^{(i)}=0
$$

(3) Update $\boldsymbol{C}$ and $\boldsymbol{F}$, and compute $J$ as follows

$$
\begin{aligned}
& \boldsymbol{C}^{(i+1)}=\boldsymbol{C}^{(i)}+\Delta C_{N N}^{(i+1)} \boldsymbol{N} \otimes \boldsymbol{N} \\
& \boldsymbol{F}^{(i+1)}=\boldsymbol{F}^{(i)}+\frac{1}{2}\left(\frac{\Delta C_{N N}^{(i+1)}}{F_{n N}^{(i)}}\right) \boldsymbol{n} \otimes \boldsymbol{N} \\
& \boldsymbol{J}^{(i+1)}=\operatorname{det} \boldsymbol{F}^{(i+1)},
\end{aligned}
$$

and increment the iteration counter $i \leftarrow i+1$.

Convergence to machine precision is achieved in only three to four iterations.

Stage 3-Finalization: The resultant values of $\boldsymbol{F}$ and $\boldsymbol{S}$ are used to assemble the left-hand-side and right-hand-side of the discrete residual equations corresponding to the weak form (9). The material tangent modulus $\boldsymbol{C}$ is modified as

$$
C_{I J K L} \leftarrow C_{I J K L}-\frac{C_{I J M N} N_{M} N_{N} N_{O} N_{P} C_{O P K L}}{N_{Q} N_{R} C_{Q R S T} N_{S} N_{T}}
$$

prior to being employed in the assembly of the left-handside matrix corresponding to the discrete variational statement (9).

Remark The surface-based solid formulation presented in this section is simple in that it only makes use of the displacement degrees of freedom. Likewise, the zero through-thickness stress condition is enforced through a straight-forward iterative algorithm. However, due to a simplified definition of the displacement variables and the deformation gradient (see Eqs. 6-8), element out-of-plane rotations generate strains and, as a result, stresses. To overcome this modeling deficiency, in the future, we plan to go to a full shell formulation with rotational degrees of freedom.
Alternatively, this issue may also be circumvented by using thee-dimensional solid modeling as in $[3,19,42,48,51]$.

\section{Numerical simulations}

\subsection{Coupled FSI formulation for vascular blood flow}

Our current moving-domain vascular flow-structure interaction computational methodology consists of the following features.

The blood is governed by the Navier-Stokes equations of incompressible flow posed on a moving domain. The Arbitrary Lagrangian-Eulerian (ALE) formulation is used (see, e.g., $[12,18])$, which is a well-suited approach for vascular blood flow applications. However, space-time fine elements were also employed for vascular FSI with great success (see, e.g., $[39,42,46,47])$. The vessel wall is modeled as a hyperelastic material in the Lagrangian description. At the interface between the blood and the elastic wall, velocity and traction compatibility conditions are assumed to hold. The motion of the fluid domain is governed by the equations of linear elasticity subject to displacement boundary conditions coming from the motion of the arterial wall.

At the discrete level, the fluid formulation makes use of the recently proposed residual-based variational multiscale method [1]. This methodology is equally applicable to turbulent and laminar flows, and is thus well-suited for our application, where the nature of the flow is not known a priori, and some regions may be turbulent while others are laminar. The Jacobian-based mesh stiffening technique is employed in which the elastic modulus of the smaller fluid elements near the solid wall is increased in proportion to the inverse of the element volume (see, e.g., [21,38,40], and [34,35,43] for more advanced mesh moving techniques). This results in fluid mesh stiffening near solid wall boundaries and, as a consequence, preservation of small elements where they are needed for accurate computation of boundary layer phenomena and the wall shear stress.

The time-dependent equations are solved using the generalized- $\alpha$ time integrator proposed in [8] for structural dynamics, and developed for fluid mechanics in [20] and FSI in [2].

The meshes for the blood volume and the vessel wall are compatible at their interface. Fluid and solid mesh compatibility is not necessary in general, however, the simulation procedures are significantly simplified as a result of this assumption: the kinematic compatibility condition is satisfied point-wise by having a unique set of degrees of freedom at the fluid-solid interface, and the traction condition holds in a weak sense.

A quasi-direct solution strategy is adopted in which the increments of the fluid and solid variables are obtained in a simultaneous fashion (see [41] for terminology and details). 


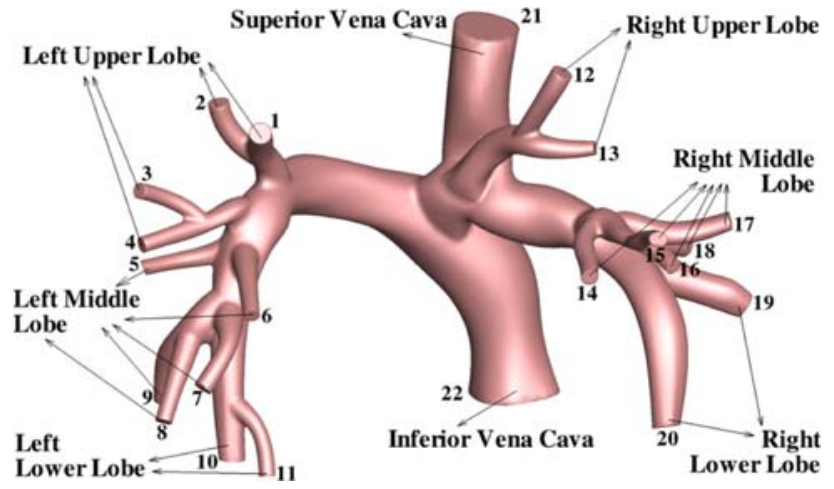

Fig. 3 Patient-specific Fontan surgery model that includes the inferior vena cava (IVC), superior vena cava (SVC), and pulmonary circulation represented by the left upper lobe (LUL), left middle lobe (LML), left lower lobe (LLL), right upper lobe (RUL), right middle lobe (RML), and right lower lobe (RLL). Image is rotated anterior to posterior for ease of viewing

The effect of the mesh motion on the fluid equations is omitted from the tangent matrix for efficiency, as advocated in [4] for cardiovascular flow-structure applications.

\subsection{Patient-specific Fontan surgery configuration computational model}

The Fontan surgery model that is used in the computations is shown in Fig. 3. The model is comprised of two inlets, corresponding to the Inferior Vena Cava (IVC) and Superior Vena Cava (SVC), for which the time-periodic flowrate is prescribed. The model also has 20 outlet branches corresponding to the pulmonary circulation. At each outlet, resistance boundary conditions are prescribed of the form

$p=C_{r} q$,

where $p$ is the pressure, $q$ is the volumetric flowrate, and $C_{r}$ is the resistance constant. The resistance data is tabulated in Table 1 and the numbering of the branches in Fig. 3 is the same as that in the table. The resistance data is chosen to match cardiac catheterization pressure data for this patient, as described below, and corresponds to case when the patient is resting, which we refer to as "rest conditions". The resistance is lowered in the case of exercise conditions, which is taken into account in the simulations presented later in this section. Note that, on the venous side, the intramural pressure is significantly lower than on the arterial side, and, as a result, the resistance boundary condition (32) does not have an ambient pressure component. As an alternative to resistance outflow boundary conditions, Windkessel (RCR) and impedance outflow boundary conditions (see, e.g., [50]) will be investigated in future studies.

Remark Because the ambient pressure in the venous circulation is very low as compared to the arterial circulation, the
Table 1 Resistance data at rest condition (in dyn s/ $\mathrm{cm}^{5}$ )

\begin{tabular}{llrllr}
\hline Tag & Name & Resistance & Tag & Name & Resistance \\
\hline 1 & LUL & 7128.27 & 12 & RUL & 4532.76 \\
2 & LUL & 8024.40 & 13 & RUL & 9693.90 \\
3 & LUL & 7426.53 & 14 & RML & 10387.80 \\
4 & LUL & 7426.35 & 15 & RML & 10062.90 \\
5 & LML & 8699.04 & 16 & RML & 11913.30 \\
6 & LML & 6870.51 & 17 & RML & 6374.43 \\
7 & LML & 11436.30 & 18 & RML & 4737.24 \\
8 & LML & 14357.70 & 19 & RLL & 3156.48 \\
9 & LML & 7777.80 & 20 & RLL & 3023.55 \\
10 & LLL & 5564.25 & 21 & SVC & - \\
11 & LLL & 15519.60 & 22 & IVC & - \\
\hline
\end{tabular}

See Fig. 3 for the numbering of the branches. For the exercise condition, the resistance values were decreased by $10 \%$

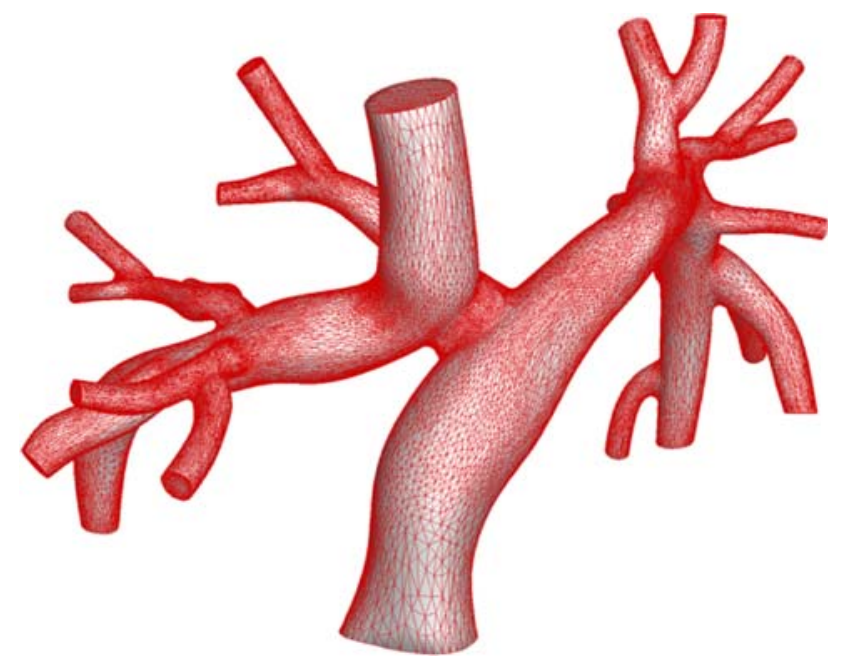

Fig. 4 Tetrahedral mesh for the Fontan surgery model

vessel configuration taken from image data may be used to approximate the reference, zero-stress configuration. However, in general, the vessel configuration coming from image data is not stress-free, which needs to be accounted for in the modeling. The reader is referred to $[37,44]$ for a method of obtaining an estimated zero pressure geometry from patientspecific image data.

We use the following material properties in our computations. The fluid density and dynamic viscosity are $1.06 \mathrm{~g} / \mathrm{cm}^{3}$ and $0.04 \mathrm{~g} / \mathrm{cm} \mathrm{s}$, respectively. The vessel wall has the density $1.00 \mathrm{~g} / \mathrm{cm}^{3}$, and shear and bulk moduli of $1.72 \times 10^{6}$ and $1.67 \times 10^{7} \mathrm{dyn} / \mathrm{cm}^{2}$, respectively.

The tetrahedral mesh of the Fontan model is shown in Fig. 4. The mesh is refined near boundary layers and regions of complex branchings based on the error indicators from a standalone fluid mechanics computation using vascular blood flow mesh adaption techniques in [32]. Although good 
Table 2 Tetrahedral mesh statistics for the Fontan surgery model

\begin{tabular}{lll}
\hline Total nodes & Total elements & Surface elements \\
\hline 200,785 & $1,010,672$ & 97,918 \\
\hline
\end{tabular}

mesh quality for standalone fluid dynamics simulation is obtained, procedures in [32] will need to be enhanced to handle coupled FSI cases. Boundary-layer mesh refinement was also used in [45] and [37]. The latter reference also included comparative results from meshes with and without boundary layer resolution.

Mesh statistics are summarized in Table 2. The mesh has over $10^{6}$ tetrahedral elements, which, in combination with boundary layer meshing and accurate numerics, ensures high-fidelity simulation results.

\subsection{Simulation results}

We simulate rest and exercise conditions, and also compare rigid and flexible wall results in each case. Exercise flow conditions correspond are generated by increasing the IVC flow rate by three times, while keeping SVC flow fixed. These values are at or slightly above the typical range for a Fontan patient found in clinical exercise data, in which on average Fontan patients are able to approximately double their cardiac index at peak exercise $[14,33]$.

The inflow flowrates as a function of time for both IVC and SVC branches are given in Fig. 5. Note that the SVC flowrate is synchronized with the heart cycle, while the IVC flowrate is synchronized with the respiratory cycle. Cardiac catheterization pressure tracings, echocardiography, and MR studies have all demonstrated that respiration significantly effects Fontan flow rates and pressures $[15,30]$. As seen with the echocardiographic tracings, quantitative real-time phase contrast MR measurements by Hjortdal et al. [15] show that flow rates in the IVC vary significantly with respiration at rest (as much as 80\%), with smaller cardiac pulsatility superimposed. Cardiac variations in the SVC were found to be small, with no significant respiratory variation. Based on this data, we impose a respiration model to model flow variations in the IVC following our previously published work [24]. This model assumes three cardiac cycles per respiratory cycle, and values of heart rate and respiratory rate are increased during exercise following the data of Hjortdal et al. Also note that the cycles are shorter, and maximum flowrate is

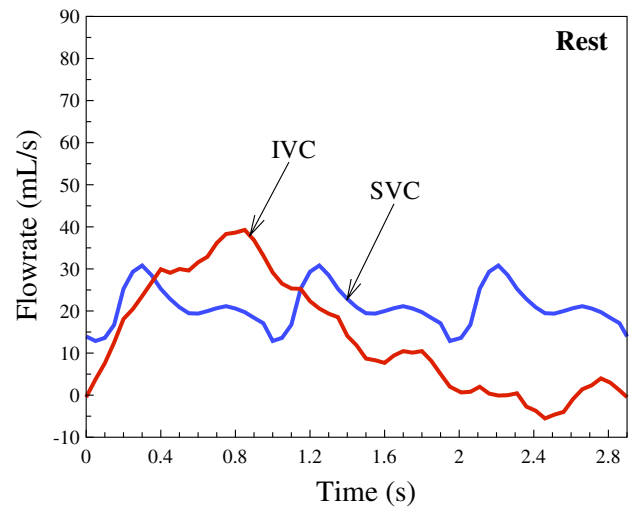

(a) Rest condition

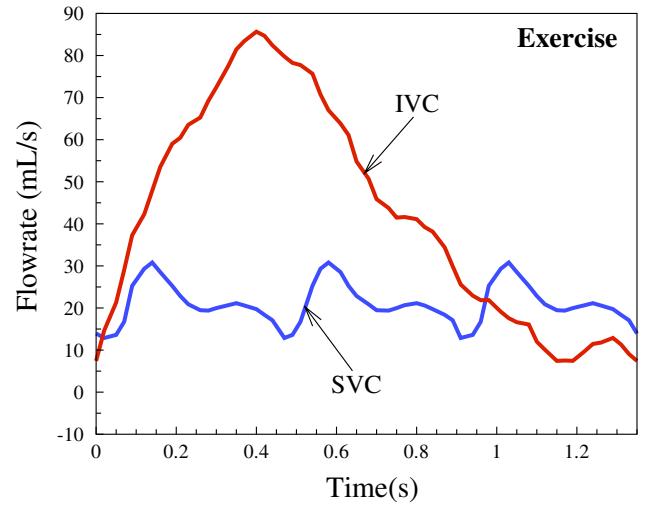

(b) Exercise condition

Fig. 5 IVC and SVC inflow flowrates for rest and exercise conditions

Fig. 6 Isosurfaces of vessel wall displacement magnitude

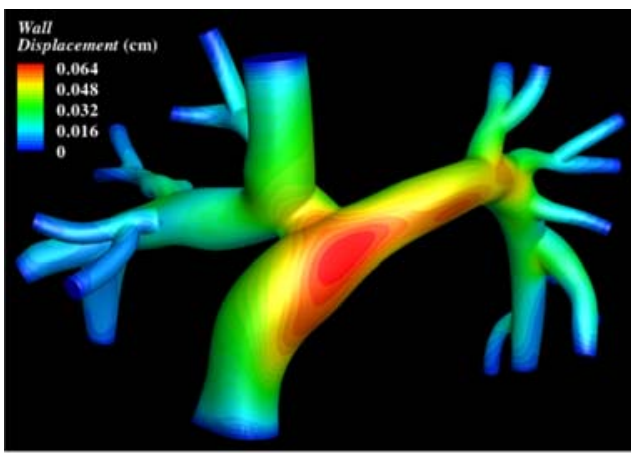

(a) Rest condition

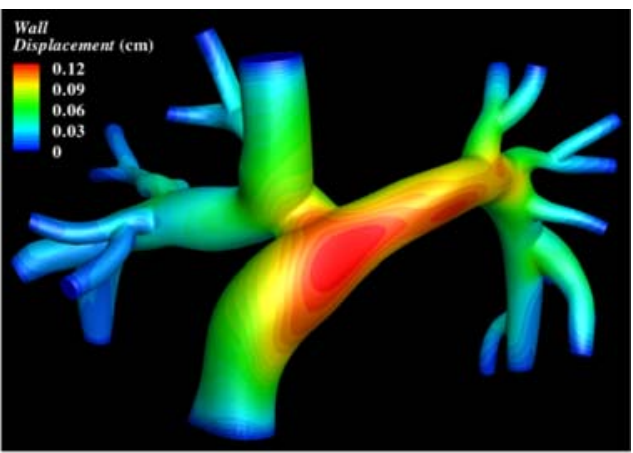

(b) Exercise condition 

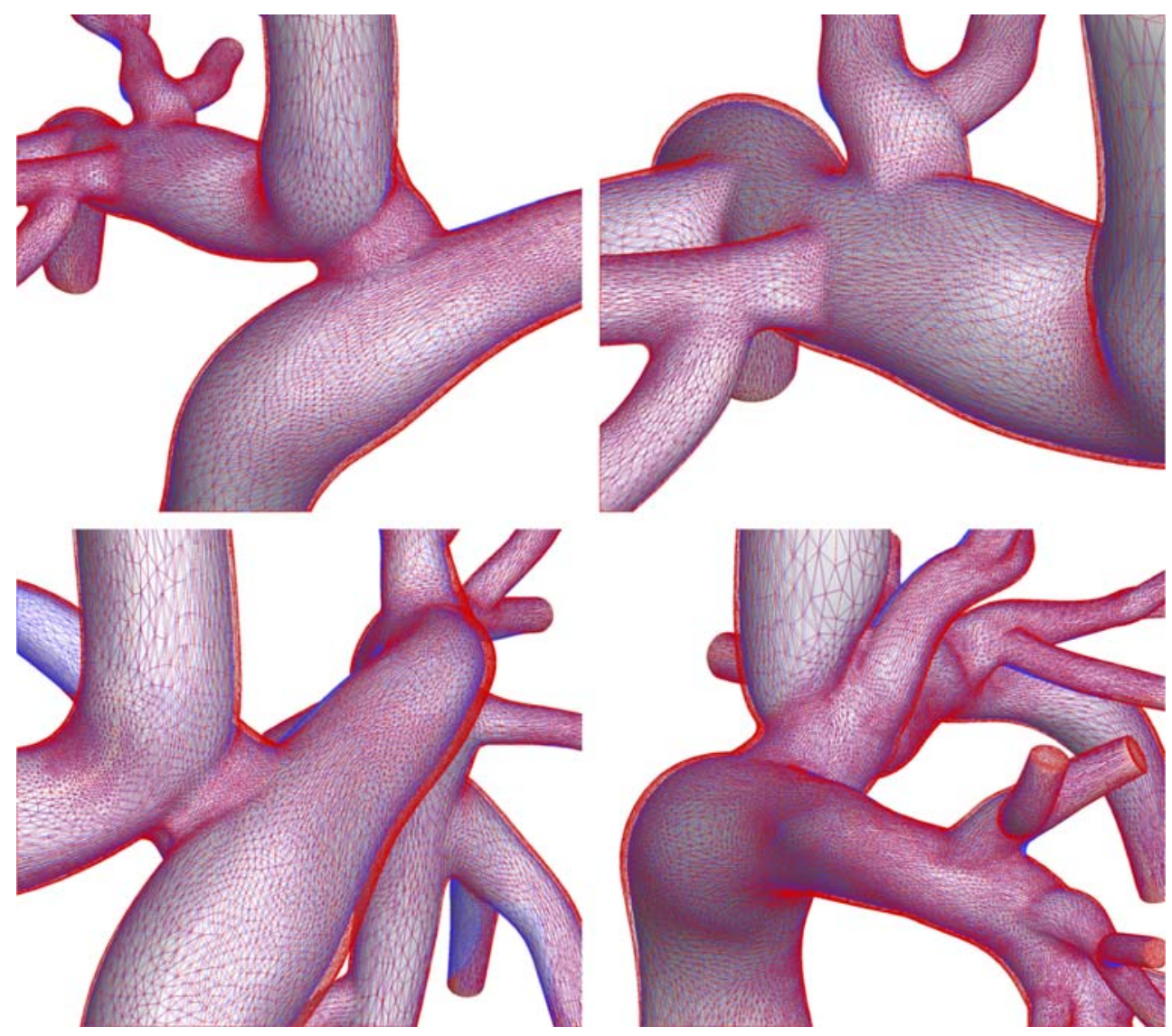

Fig. 7 Comparison of the Fontan model configurations at high systole and low diastole

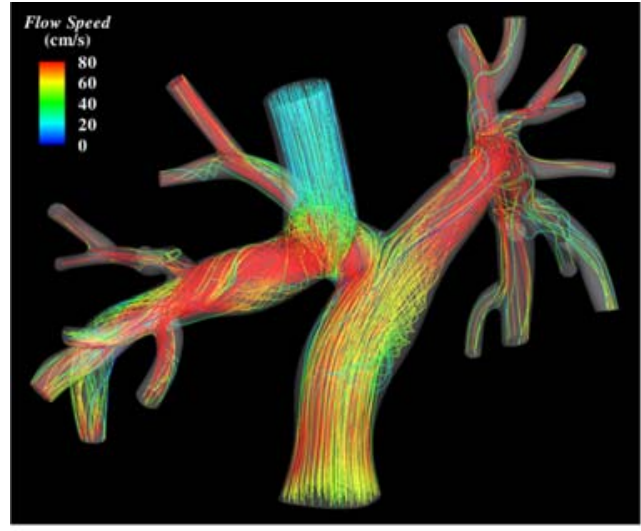

(a) Rigid wall

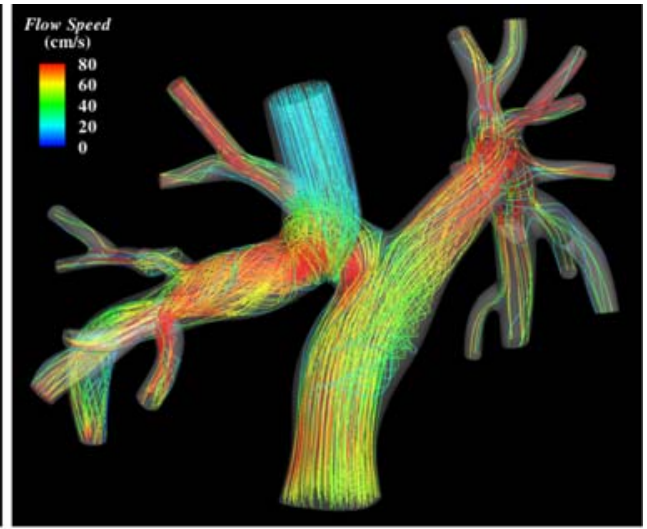

(b) Flexible wall

Fig. 8 Comparison of blood velocity streamlines at peak flowrate for exercise conditions

significantly higher for the exercise condition case. Resistance data provided in Table 1 corresponds to the case of rest conditions. Downstream resistance values were chosen to match patient-specific cardiac catheterization pressure in the IVC and SVC. The LPA/RPA flow split of 45/55 was prescribed. On each side (LPA and RPA), flow was distrib- uted amongst the pulmonary outlets by grouping them into upper, middle, and lower lobes. Resistances were chosen to distribute $20 \%$ of the flow to the upper lobe and $40 \%$ each to the middle and lower lobes. These values were based on the assumption that each of the ten major lung segments receives equal flow. Within each lobe group, flow was distributed 


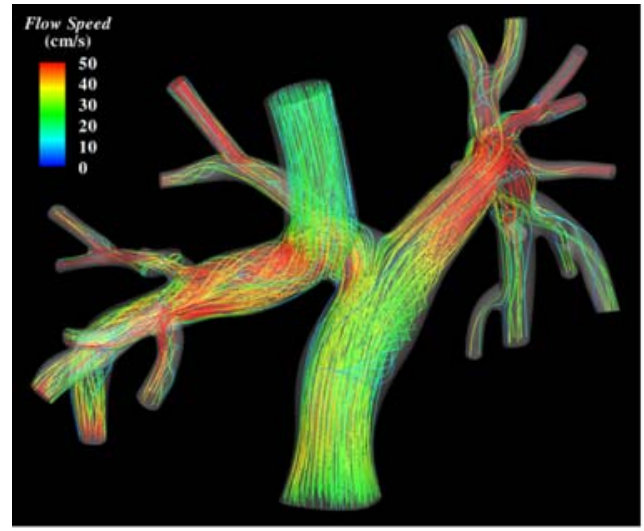

(a) Rigid wall

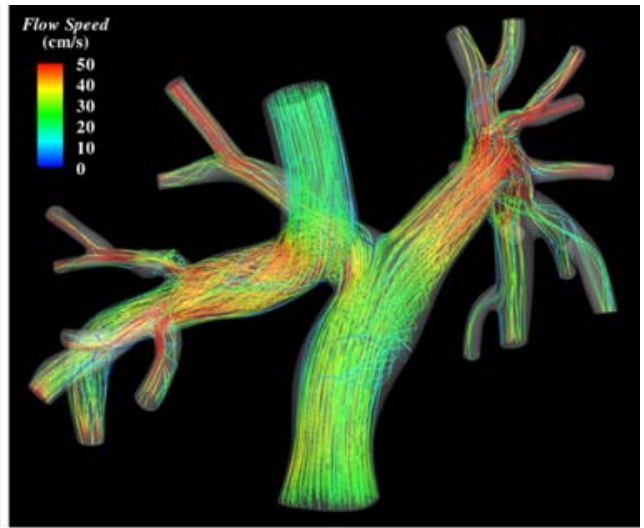

(b) Flexible wall

Fig. 9 Comparison of blood velocity streamlines at peak flowrate for rest conditions

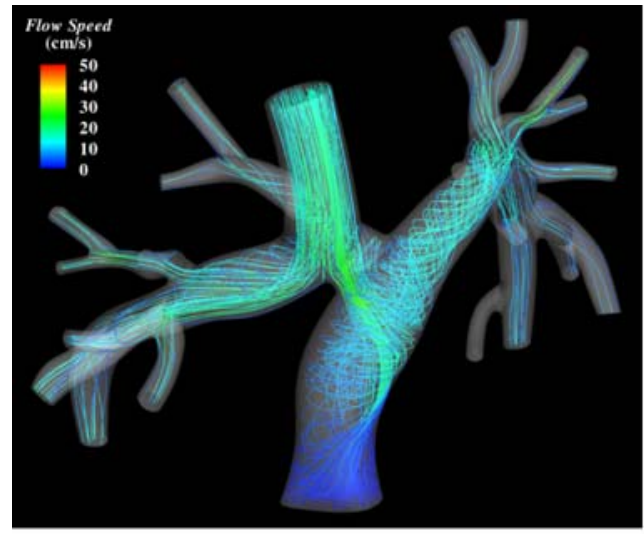

(a) Rest conditions

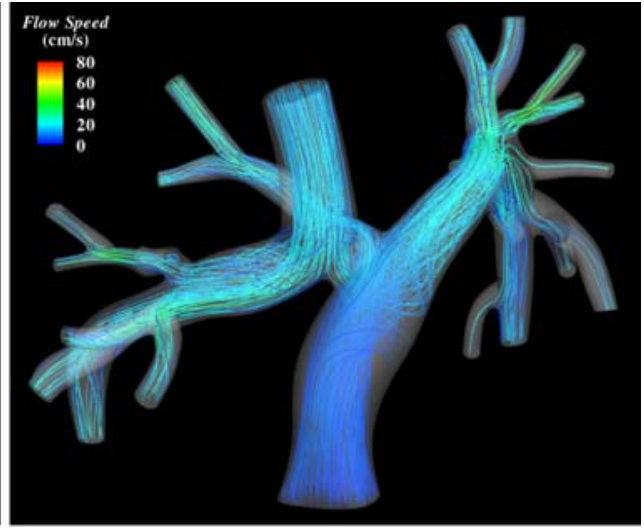

(b) Exercise conditions

Fig. 10 Blood velocity streamlines at low flowrate

Fig. 11 Comparison of wall shear stress at rest conditions

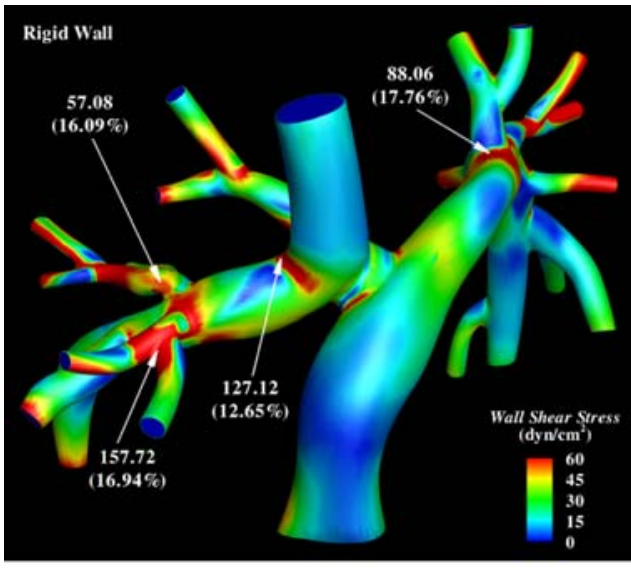

(a) Rigid wall

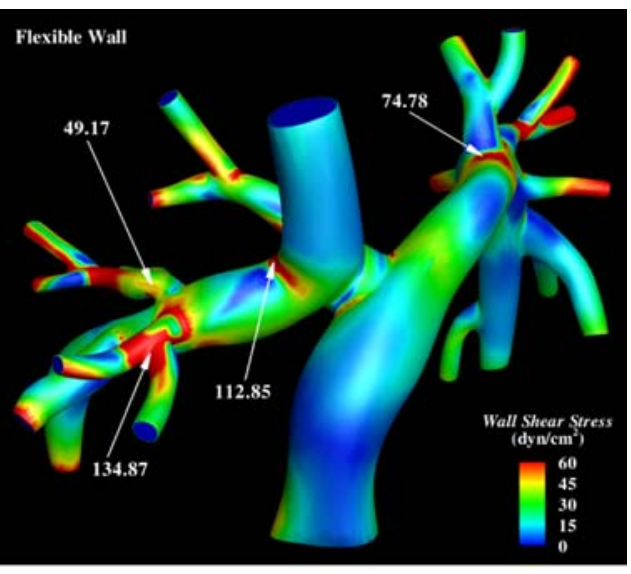

(b) Flexible wall according to the outlet areas. An initial steady simulation was run to verify that catheterization pressure data was matched within $0.5 \mathrm{mmHg}$ in the IVC and SVC. For exercise conditions, resistivity of the vessels decreases uniformly to $90 \%$ of the original values based on clinical observations that pul- monary vascular resistance (PVR) in both normal children and Fontan patients decreases with exercise [23,33].

Figure 6 shows isosurfaces of the vessel wall displacement magnitude for rest and exercise simulations. The inflow flowrate is higher for the case of exercise conditions leading to 
Fig. 12 Comparison of wall shear stress at exercise conditions

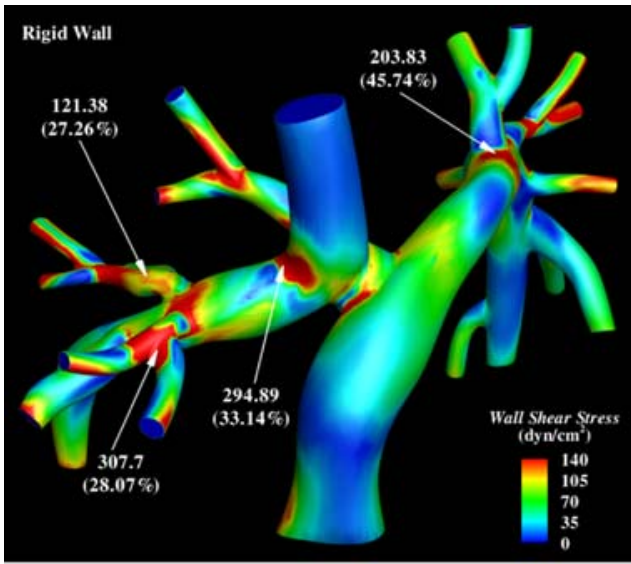

(a) Rigid wall

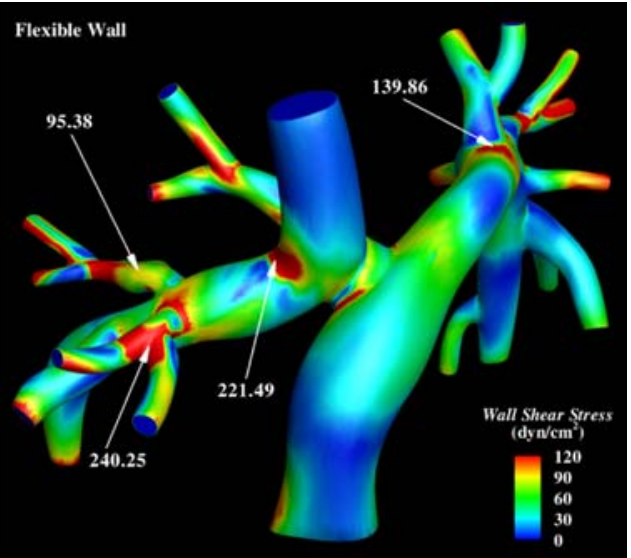

(b) Flexible wall

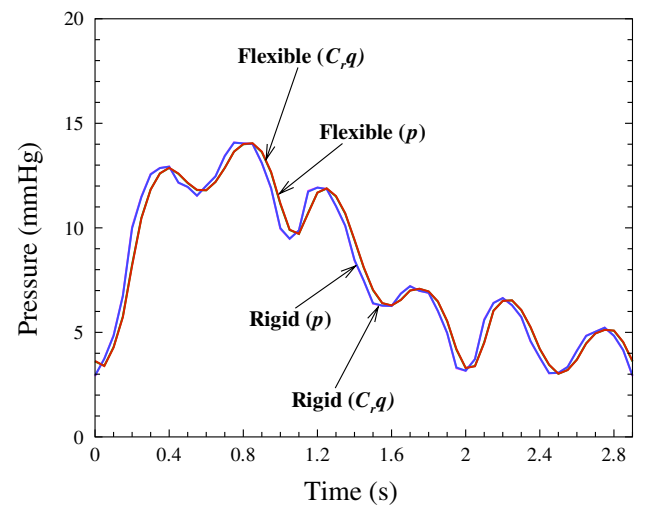

(a) Outlet 2

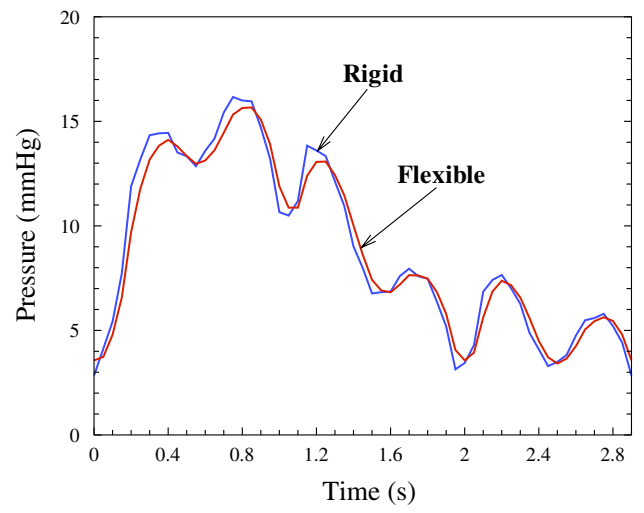

(c) SVC

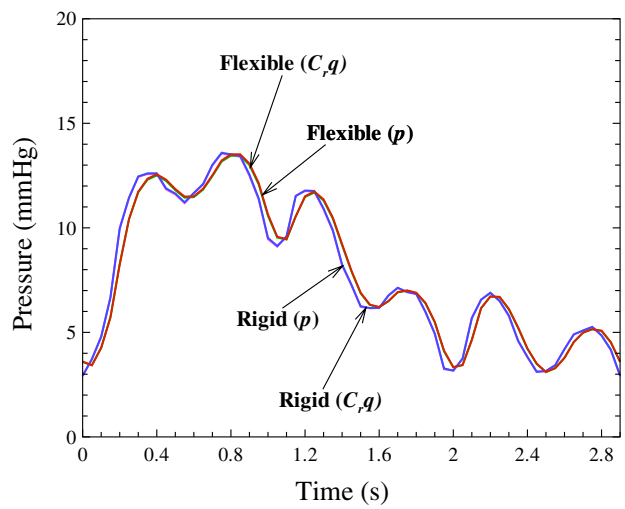

(b) Outlet 17

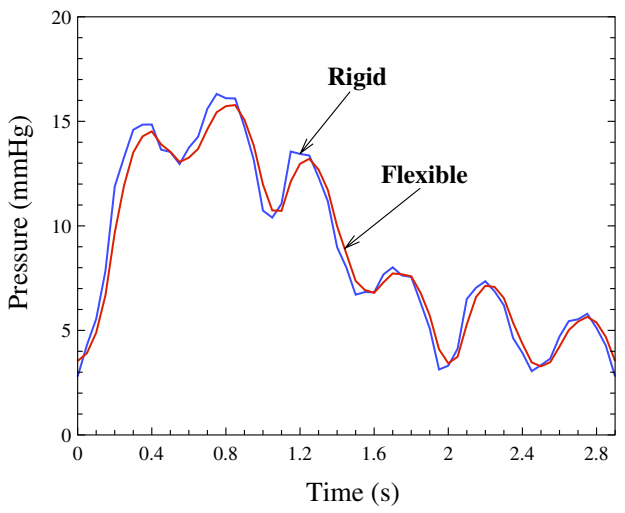

(d) IVC

Fig. 13 Comparison of blood pressure at IVS, SVC, and selected outlets at rest conditions

increased levels of intramural pressure and, as a result, larger magnitude of wall displacement. We zoom on the different parts of the model and compare the configurations at peak systole and low diastole in Fig. 7. We can see from the figure that the relative displacement between the two configurations is quite moderate.

Figure 8 shows the blood flow velocity streamlines at peak systole for the exercise condition simulation, comparing rigid and flexible results. The rigid wall case gives an over-prediction of the instantaneous flow speed with respect to the flexible case. There are also some differences in the streamline patterns, especially in the regions of complex vessel branchings. Velocity streamlines at rest conditions are compared in Fig. 9. Although the flow speed is again overpredicted by the rigid wall simulation, the differences in the streamline patterns are less pronounced. Flow streamlines at low flowrate comparing rest and exercise conditions are shown in Fig. 10. Under rest conditions, due to flow reversal 


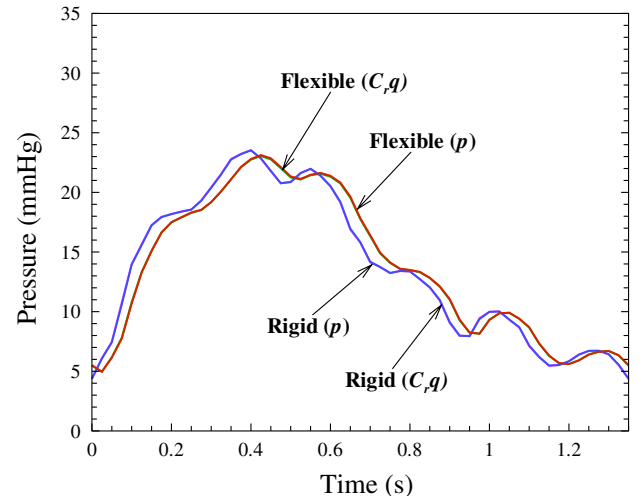

(a) Outlet 2

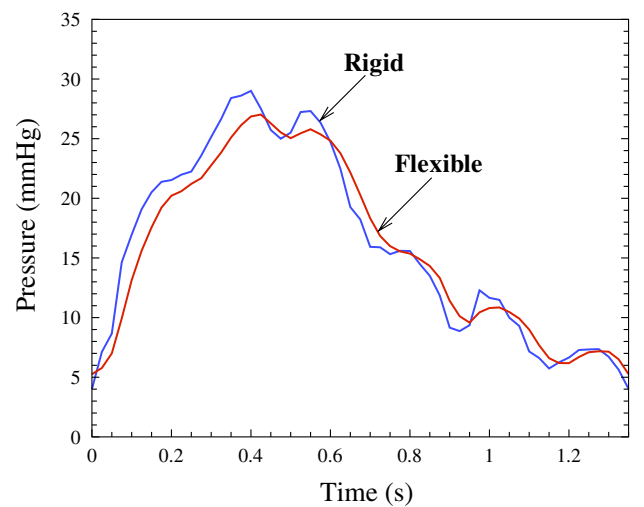

(c) SVC

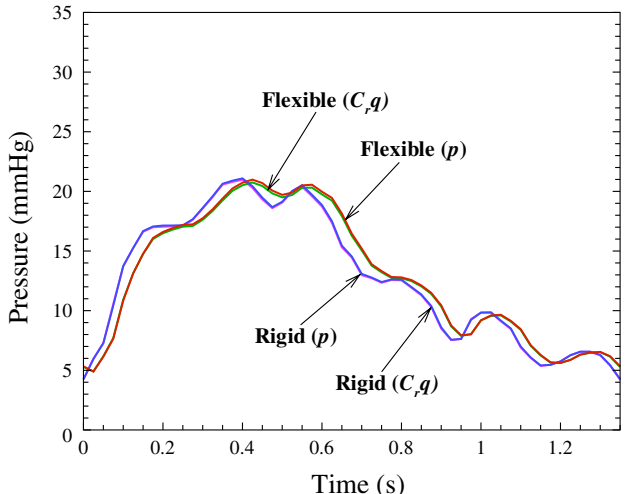

(b) Outlet 17

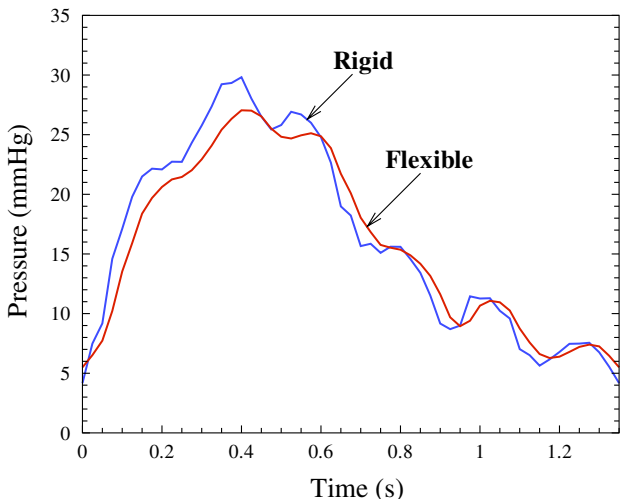

(d) IVC

Fig. 14 Comparison of blood pressure at IVS, SVC, and selected outlets at exercise conditions

during the later parts of the respiratory cycle, a swirl-like feature develops near the inflow of the IVC giving rise to helical flow patters in the branch. This feature is not present for the exercise condition case.

We next examine the wall shear stress (WSS) and compare the results of rigid wall and flexible wall simulations. In Fig. 11, the rest conditions are compared at peak flow, while Fig. 12 shows a comparison for the exercise case. The overall distribution of WSS is similar and WSS highs and lows tend to concentrate near the arterial branchings. A closer examination of the WSS data revealed that in the case of rest conditions, the WSS for the rigid wall simulation over-predicts that of the flexible wall by as much $17 \%$ in the case of rest conditions and by as much as $45 \%$ in the case of exercise conditions. The data was taken at several discrete locations on the arterial wall and the locations are indicated by arrows in the figures. This data, as well as the flow streamline comparison presented above, clearly shows that flexible wall modeling is important for Fontan surgery simulations and has a greater effect on the outcomes of the simulations in the case of exercise conditions.

Computed pressure time histories for SVC, IVC, and selected outlet branches are shown in Figs. 13 and 14. In all cases there is a distinct pressure time lag between rigid and flexible simulation results. Furthermore, the flexible wall assumption produces a smoothing effect on the pressure output. In both rest and exercise cases, the pressure peak is always higher for the the rigid wall simulation and the overprediction is greater for the exercise conditions simulation, just as in the case of the WSS.

Finally, in Figs. 13 and 14, the outlet pressure data is presented in two ways: $i$. The pressure field is taken directly and averaged over the outlet cross-sections; ii. The flowrate is computed through the outlets and multiplied by the corresponding resistance constant (see equation (32)). The figures show no visible differences between the two quantities. This indicates that resistance boundary conditions, although imposed only weakly in the discrete formulation, actually hold in a nearly strong sense. (For the numerical formulation and implementation details of the weak enforcement of pressure-flow boundary conditions, see, e.g., $[2,4]$.)

\section{Conclusions}

We applied computational fluid-structure interaction analysis to the simulation of a patient-specific Fontan surgery configuration. Tetrahedral meshes that are refined near boundary layers and regions of complex blood vessel branching are employed for the fluid mechanics part of 
the simulation. Structural discretization makes use of the resultant triangulation of the blood vessel surface. A simple structural model with only displacement degrees of freedom and a hyperelastic constitutive law is proposed, for which a zero through-thickness stress condition is enforced using an integration-point-level Newton iteration algorithm. Variable blood vessel wall thickness is assumed in the simulations, which is prescribed using a newly developed reconstruction technique based on the Laplace partial differential equation. Despite the ad hoc nature of the approach, it leads to physiologically realistic vessel wall thickness distributions for patient-specific vascular models, independent of their geometric complexity.

Simulation results for a patient-specific Fontan model show that flexible wall modeling has an important effect on quantities of hemodynamic interest, and thus cannot be neglected for this class of problems. In particular, blood flow patterns differ for rigid and flexible wall simulations, and the wall shear stress and pressures are over-predicted when the rigid wall assumption is employed. The differences in these quantities are more pronounced for the exercise conditions simulations, for which flowrates and pressures are higher, and blood flow velocities have greater spatial and temporal variability.

Limitations of this work include a lack of patient specific clinical data on pulmonary flow distribution and exercise hemodynamics (flow waveforms and resistance values). These limitations will be addressed in future clinical studies. Another limitation is the lack of material property data for vasculature on the venous side. Future work should examine the effects of uncertainties in these quantities on simulation outputs.

Acknowledgments We wish to thank the Texas Advanced Computing Center (TACC) at the University of Texas at Austin for providing HPC resources that have contributed to the research results reported within this paper. Support of Teragrid Grant No. MCAD7S032 is gratefully acknowledged. Alison Marsden was supported by a Burroughs Wellcome Fund Career Award at the Scientific Interface, and by an American Heart Association Beginning Grant in Aid award. We would also like to thank Jeff Feinstein for his valuable input on the clinical relevance of the reported simulations.

Open Access This article is distributed under the terms of the Creative Commons Attribution Noncommercial License which permits any noncommercial use, distribution, and reproduction in any medium, provided the original author(s) and source are credited.

\section{References}

1. Bazilevs Y, Calo VM, Cottrel JA, Hughes TJR, Reali A, Scovazzi G (2007) Variational multiscale residual-based turbulence modeling for large eddy simulation of incompressible flows. Comput Methods Appl Mech Eng 197:173-201
2. Bazilevs Y, Calo VM, Hughes TJR, Zhang Y (2008) Isogeometric fluid-structure interaction: theory, algorithms, and computations. Comput Mech 43:3-37

3. Bazilevs Y, Calo VM, Zhang Y, Hughes TJR (2006) Isogeometric fluid-structure interaction analysis with applications to arterial blood flow. Comput Mech 38:310-322

4. Bazilevs Y, Gohean JR, Hughes TJR, Moser RD, Zhang Y (2009) Patient-specific isogeometric fluid-structure interaction analysis of thoracic aortic blood flow due to implantation of the Jarvik 2000 left ventricular assist device. Comput Methods Appl Mech Eng. doi:10.1016/j.cma.2009.04.015

5. Bazilevs Y, Hsu M-C, Zhang Y, Wang W, Liang X, Kvamsdal T, Brekken R, Isaksen JG (2009) A fully-coupled fluid-structure interaction simulation of cerebral aneurysms. Comput Mech, In the same issue

6. Bischoff M, Wall WA, Bletzinger K-U, Ramm E (2004) Models and finite elements for thin-walled structures. In: Stein E, de Borst R, Hughes TJR (eds) Encyclopedia of computational mechanics, vol 2, Solids, structures and coupled problems, chap 3. Wiley

7. Bove EL, de Leval MR, Migliavacca F, Guadagni G, Dubini G (2003) Computational fluid dynamics in the evaluation of hemodynamic performance of cavopulmonary connections after the Norwood procedure for hypoplastic left heart syndrome. J Thorac Cardiovasc Surg 126:1040-1047

8. Chung J, Hulbert GM (1993) A time integration algorithm for structural dynamics with improved numerical dissipation: the generalized- $\alpha$ method. J Appl Mech 60:371-375

9. de Leval MR, Dubini G, Migliavacca F, Jalali H, camporini G, Redington A, Pietrabissa R (1996) Use of computational fluid dynamics in the design of surgical procedures: application to the study of competitive flows in cavo-pulmonary connections. J Thorac Cardiovasc Surg 111(3):502-513

10. Dubini G, de Leval MR, Pietrabissa R, Montevecchi FM, Fumero R (1996) A numerical fluid mechanical study of repaired congenital heart defects: application to the total cavopulmonary connection. $\mathrm{J}$ Biomech 29(1):111-121

11. Ensley AE, Ramuzat A, Healy TM, Chatzimavroudis GP, Lucas C, Sharma S, Pettigrew R, Yoganathan AP (2000) Fluid mechanic assessment of the total cavopulmonary connection using magnetic resonance phase velocity mapping and digital particle image velocimetry. Ann Biomed Eng 28:1172-1183

12. Farhat C, Geuzaine P, Grandmont C (2001) The discrete geometric conservation law and the nonlinear stability of ALE schemes for the solution of flow problems on moving grids. J Comput Phys 174(2):669-694

13. Fontan F, Baudet E (1971) Surgical repair of tricuspid atresia. Thorax 26:240-248

14. Giardini A, Balducci A, Specchia S, Gaetano G, Bonvicini M, Picchio FM (2008) Effect of sildenafil on haemodynamic response to exercise capacity in fontan patients. Eur Heart J 29:1681-1687

15. Hjortdal VE, Emmertsen K, Stenbog E, Frund T, Rahbek Schmidt M, Kromann O, Sorensen K, Pedersen EM (2003) Effects of exercise and respiration on blood flow in total cavopulmonary connection: a real-time magnetic resonance flow study. Circulation 108:1227-1231

16. Holzapfel GA (2000) Nonlinear solid mechanics, a continuum approach for engineering. Wiley, Chichester

17. Hughes TJR (2000) The finite element method: linear static and dynamic finite element analysis. Dover Publications, Mineola

18. Hughes TJR, Liu WK, Zimmermann TK (1981) LagrangianEulerian finite element formulation for incompressible viscous flows. Comput Methods Appl Mech Eng 29:329-349

19. Isaksen JG, Bazilevs Y, Kvamsdal T, Zhang Y, Kaspersen JH, Waterloo K, Romner B, Ingebrigtsen T (2008) Determination of wall tension in cerebral artery aneurysms by numerical simulation. Stroke 39:3172-3178 
20. Jansen KE, Whiting CH, Hulbert GM (1999) A generalized- $\alpha$ method for integrating the filtered Navier-Stokes equations with a stabilized finite element method. Comput Methods Appl Mech Eng 190:305-319

21. Johnson AA, Tezduyar TE (1994) Mesh update strategies in parallel finite element computations of flow problems with moving boundaries and interfaces. Comput Methods Appl Mech Eng 119:73-94

22. Khunatorn Y, Mahalingam S, DeGroff CG, Shandas R (2002) Influence of connection geometry and SVC-IVC flow rate ratio on flow structures within the total cavopulmonary connection: a numerical study. J Biomech Eng Trans ASME 124:364-377

23. Kulik TJ, Bass JL, Fuhrman BP, Moller JH, Lock JE (1983) Exercise induced pulmonary vasoconstriction. Br Heart J 50:59-64

24. Marsden AL, Vignon-Clementel IE, Chan F, Feinstein JA, Taylor CA (2007) Effects of exercise and respiration on hemodynamic efficiency in CFD simulations of the total cavopulmonary connection. Ann Biomed Eng 35(2):250-263

25. Marsden AL, Bernstein AD, Reddy VM, Shadden S, Spilker R, Chan FP, Taylor CA, Feinstein JA (2009) Evaluation of a novel Y-shaped extracardiac fontan baffle using computational fluid dynamics. J Thorac Cardiovasc Surg, To appear

26. Marsden AL, Vignon-Clementel IE, Chan F, Feinstein JA, Taylor CA (2007) Effects of exercise and respiration on hemodynamic efficiency in CFD simulations of the total cavopulmonary connection. Ann Biomed Eng 35:250-263

27. Masters JC, Ketner M, Bleiweis MS, Mill M, Yoganathan A, Lucas CL (2004) The effect of incorporating vessel compliance in a computational model of blood flow in a total cavopulmonary connection (tcpc) with caval centerline offset. J Biomech Eng 126:709-713

28. Migliavacca F, Dubini G, Bove EL, de Leval MR (2003) Computational fluid dynamics simulations in realistic 3-D geometries of the total cavopulmonary anastomosis: the influence of the inferior caval anastomosis. J Biomech Eng 125:805-813

29. Migliavacca F, Dubini G, Pietrabissa R, de Leval MR (1997) Computational transient simulations with varying degree and shape of pulmonic stenosis in models of the bidirectional cavopulmonary anastomosis. Med Eng Phys 19:394-403

30. Pedersen EM, Stenbog EV, Frund T, Houlind K, Kromann O, Sorensen KE, Emmertsen K, Hjortdal VE (2002) Flow during exercise in the total cavopulmonary connection measured by magnetic resonance velocity mapping. Heart 87:554-558

31. Petrossian E, Reddy VM, Collins KK, Culbertson CB, MacDonald MJ, Lamberti JJ, Reinhartz O, Mainwaring RD, Francis PD, Malhotra SP, Gremmels DB, Suleman S, Hanley FL (2006) The extracardiac conduit Fontan operation using minimal approach extracorporeal circulation: early and midterm outcomes. J Thorac Cardiovasc Surg 132(5):1054-1063

32. Sahni O, Muller J, Jansen KE, Shephard MS, Taylor CA (2006) Efficient anisotropic adaptive discretization of the cardiovascular system. Comput Methods Appl Mech Eng 195:56345655

33. Shachar GB, Fuhrman BP, Wang Y, Lucas RVJr, Lock JE (1982) Rest and exercise hemodynamics after the fontan procedure. Circulation 65:1043-1048

34. Stein K, Tezduyar T, Benney R (2003) Mesh moving techniques for fluid-structure interactions with large displacements. J Appl Mech 70:58-63

35. Stein K, Tezduyar TE, Benney R (2004) Automatic mesh update with the solid-extension mesh moving technique. Comput Methods Appl Mech Eng 193:2019-2032

36. Takizawa K, Christopher J, Moorman C, Martin J, Purdue J, McPhail T, Chen PR, Warren J, Tezduyar TE (2009) Space-time finite element computation of arterial FSI with patient-specific data. In: Schrefler B, Onate E, Papadrakakis M (eds) Computational methods for coupled problems in science and engineering, coupled problems 2009

37. Takizawa K, Christopher J, Tezduyar TE, Sathe S (2009) Spacetime finite element computation of arterial fluid-structure interactions with patient-specific data. Commun Numer Methods Eng, published online. doi:10.1002/cnm.1241

38. Tezduyar TE, Aliabadi S, Behr M, Johnson A, Mittal S (1993) Parallel finite element computation of 3D flows. Computer 26:27-36

39. Tezduyar TE (2003) Computation of moving boundaries and interfaces and stabilization parameters. Int J Numer Methods Fluids 43:555-575

40. Tezduyar TE, Behr M, Mittal S, Johnson AA (1992) Computation of unsteady incompressible flows with the stabilized finite element methods - space-time formulations, iterative strategies and massively parallel implementations. In: New methods in transient analysis, PVP-Vol. 246/ AMD-Vol. 143, pp 7-24. ASME, New York

41. Tezduyar TE, Sathe S (2007) Modelling of fluid-structure interactions with the space-time finite elements: solution techniques. Int J Numer Methods Fluids 54:855-900

42. Tezduyar TE, Sathe S, Cragin T, Nanna B, Conklin BS, Pausewang J, Schwaab M (2007) Modelling of fluid-structure interactions with the space-time finite elements: arterial fluid mechanics. Int J Numer Methods Fluids 54:901-922

43. Tezduyar TE, Sathe S, Keedy R, Stein K (2006) Space-time finite element techniques for computation of fluid-structure interactions. Comput Methods Appl Mech Eng 195:2002-2027

44. Tezduyar TE, Sathe S, Schwaab M, Conklin BS (2008) Arterial fluid mechanics modeling with the stabilized space-time fluid-structure interaction technique. Int J Numer Methods Fluids 57:601-629

45. Tezduyar TE, Schwaab M, Sathe S (2008) Sequentially-coupled arterial fluid-structure interaction (SCAFSI) technique. Comput Methods Appl Mech Eng, published online. doi:10.1016/j.cma. 2008.05.024

46. Torii R, Oshima M, Kobayashi T, Takagi K, Tezduyar TE (2006) Computer modeling of cardiovascular fluid-structure interactions with the deforming-spatial-domain/stabilized space-time formulation. Comput Methods Appl Mech Eng 195:1885-1895

47. Torii R, Oshima M, Kobayashi T, Takagi K, Tezduyar TE (2006) Fluid-structure interaction modeling of aneurysmal conditions with high and normal blood pressures. Comput Mech 38:482-490

48. Torii R, Oshima M, Kobayashi T, Takagi K, Tezduyar TE (2008) Fluid-structure interaction modeling of a patient-specific cerebral aneurysm: influence of structural modeling. Comput Mech 43:151159

49. Torii R, Oshima M, Kobayashi T, Takagi K, Tezduyar TE (2009) Influence of wall thickness on fluid-structure interaction computations of cerebral aneurysms. Commun Numer Methods Eng, published online. doi:10.1002/cnm.1289

50. Vignon-Clementel IE, Figueroa CA, Jansen KE, Taylor CA (2006) Outflow boundary conditions for three-dimensional finite element modeling of blood flow and pressure in arteries. Comput Methods Appl Mech Eng 195:3776-3796

51. Zhang Y, Wang W, Liang X, Bazilevs Y, Hsu M-C, Kvamsdal T, Brekken R, Isaksen JG (2009) High-fidelity tetrahedral mesh generation from medical imaging data for fluid-structure interaction analysis of cerebral aneurysms. Comput Model Eng Sci 42:131149 